\title{
LIPID TRAFFIC IN ANIMAL CELLS
}

\author{
Gerrit van Meer
}

Department of Cell Biology, Medical School, University of Utrecht, Utrecht, The Netherlands

\begin{abstract}
CONTENTS
SCOPE

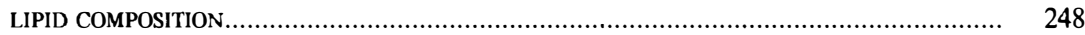

General Remarks ..................................................................................... 248

Lipid Compositions along the Endo- and Exocytic Pathways ................................. 249

Lipid Composition of Organelles Not Connected by Vesicular Traffic ......................... 251

Quantitative Distribution of Lipids between the Various Organelles .............................. 251

Transmembrane Lipid Asymmetry .......................................................................... 252

LIPID TRAFFIC AND SORTING ............................................................................. 254

Generation and Maintenance of the Heterogeneous Distribution of Sphingolipids ..... 254

Generation and Maintenance of the Heterogeneous Distribution of Cholesterol.......... 260

Generation and Maintenance of the Heterogeneous Distribution of Glycerophospholipids .................................................................................................... 264

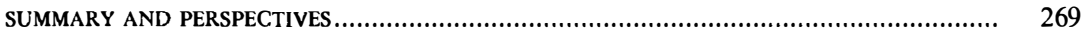

\section{SCOPE}

The membranes of the various intracellular organelles possess unique lipid compositions. Yet most of the lipids are synthesized in the endoplasmic reticulum (ER). ${ }^{1}$ This implies that lipids are transported and that specificity exists in the traffic routes to the various organelles: in other words, lipids are sorted. For various reasons cellular lipid research lags behind that of the proteins. As a consequence, a large part of the basic characterization

'Abbreviations: C6-NBD-, N-6[7-nitro-2,1,3-benzoxadiazol-4-yl] aminocaproyl-; CL, cardiolipin; ER, Endoplasmic Reticulum; GSL(s), glycosphingolipid(s); kd, kilodalton; LBPA, lysobisphosphatidic acid; PC, phosphatidylcholine; PE, phosphatidylethanolamine; PG, phosphatidylglycerol; PI, phosphatidylinositol; PS, phosphatidylserine; SCP-2, sterol carrier protein 2; SPH, sphingomyelin. 
still has to be done. This review summarizes our knowledge on lipid traffic, seeks to locate the sites of sorting events, and provides some ideas on the mechanisms of lipid sorting.

Although lipids can be transported in the same way as membrane proteins, by carrier vesicles, lipid traffic is special in a number of respects. (a) As lipid molecules are much smaller than proteins $\left(\mathrm{M}_{\mathrm{r}}\right.$ less than $\left.1 \mathrm{k}\right)$, a membrane contains 10-100 times more lipid molecules than proteins. (b) The lateral diffusion (and intermixing) of lipids is ten times faster than that of the proteins. (c) The basic structure of a membrane is the lipid bilayer. Therefore, in terms of composition and transport every membrane has to be regarded as a cytoplasmic and an exoplasmic pool of lipids that may or may not communicate. $(d)$ The differences in lipid composition between the organelles are not absolute. As all organelles possess more or less the same lipid classes, it is the ratio between these classes and their molecular species that is unique. $(e)$ In addition to the ER, most organelles have some limited lipid biosynthesizing activity and examples of metabolic interconversion of one lipid into another are known for almost every organelle. $(f)$ Proteins have been purified from cytosol that, in vitro at least, are capable of transferring most types of lipid to and from most intracellular membranes.

So the question is how the dynamic interplay between local synthesis and modification, the various modes of lipid traffic, and the inherent sorting potential of various lipids results in a stable intracellular lipid heterogeneity that is remarkably uniform between many functionally different cell types. Cellular lipid traffic will undoubtedly be more complicated than the schemes provided here. However, the complications may turn out to be variations on a common theme.

\section{LIPID COMPOSITION}

\section{General Remarks}

Phospholipids constitute the main lipid mass of eukaryotic cell membranes. In addition, membranes may contain bulk quantities of cholesterol and glycosphingolipids (GSLs). The content of the latter two lipids ranges from essentially zero as in mitochondria to equimolar amounts with the phospholipids as in the apical plasma membrane of epithelial cells (see below). Glycoglycerolipids, triglycerides, cholesterol esters, and intermediates in lipid metabolism like di- and monoglycerides, ceramides, lysophospholipids, and free fatty acids are minor membrane components under normal conditions. In contrast, the amount of dolichol, a hitherto neglected membrane lipid, may turn out to be significant (Chojnacki \& Dallner 1988). 
An evaluation of organellar lipid compositions from the literature meets a number of complications. (a) Compositional data are often incomplete. In order to be able to reconstruct a membrane, one needs the total lipid composition, expressed in terms of concentration, mol/surface area, or percent of total lipids. However, GSLs (and dolichol), for example, are frequently omitted from the analysis altogether, simply because of difficulties of quantitation $(b)$ Compositional studies have mostly been performed on membrane fractions, with the inherent difficulties of mutual contamination and incomplete yields. As an additional complication, membranes have frequently been contaminated by the lipid containing contents of organelles, e.g. secretory or endocytosed lipoproteins and the content fraction of multivesicular endosomes or lysosomes. Finally, some organelles, like endosomes, have only recently been appreciated as structurally distinct compartments and still more organelles may await discovery. (c) As a further complication, some organelles are differentiated into subcompartments of different composition, for example, the smooth and rough ER. (d) Finally, for studying lipid traffic one would like to specify the lipid composition of each of the two leaflets of the lipid bilayer. In most cases this information is not available.

\section{Lipid Compositions along the Endo- and Exocytic Pathways}

In animal eukaryotes all membrane and secretory proteins, with the exception of those destined for the mitochondria and peroxisomes, pass through the ER and are dispatched from the ER to their destination by carrier vesicles. The elucidation of the routing of these proteins has led to a model of endo- and exocytic pathways and their interconnections as illustrated in a simplified form in Figure 1.

The lipid compositions of the ER and the plasma membrane are strikingly different (Table 1). Plasma membranes are typically enriched in cholesterol, also the phospholipids SPH and PS, whereas PC is decreased. The plasma membrane invariably contains GSLs, which are thought to be absent from the ER. Extreme levels of GSLs occur in the apical plasma membrane of epithelial cells, where the GSLs constitute up to $33 \mathrm{~mol} \%$ (see below) of the total lipids. The composition of the Golgi complex is intermediate between that of the ER and the plasma membrane (Zambrano et al 1975). In fact, a compositional gradient may exist over the Golgi stack. $^{2}$ Trans Golgi cisternae appear to possess a higher concentration of

\footnotetext{
${ }^{2}$ The Golgi complex is taken to consist of at least two compartments, the cis Golgi in vesicular equilibrium with the ER (Pelham, this volume), and the "trans Golgi network" the trans most cisterna of the Golgi complex that is in vesicular equilibrium with endosomes and plasma membrane, as reviewed by Griffiths \& Simons (1986). The obvious fact that the stack contains more cisternae (Farquhar 1985) does not affect the present argument.
} 


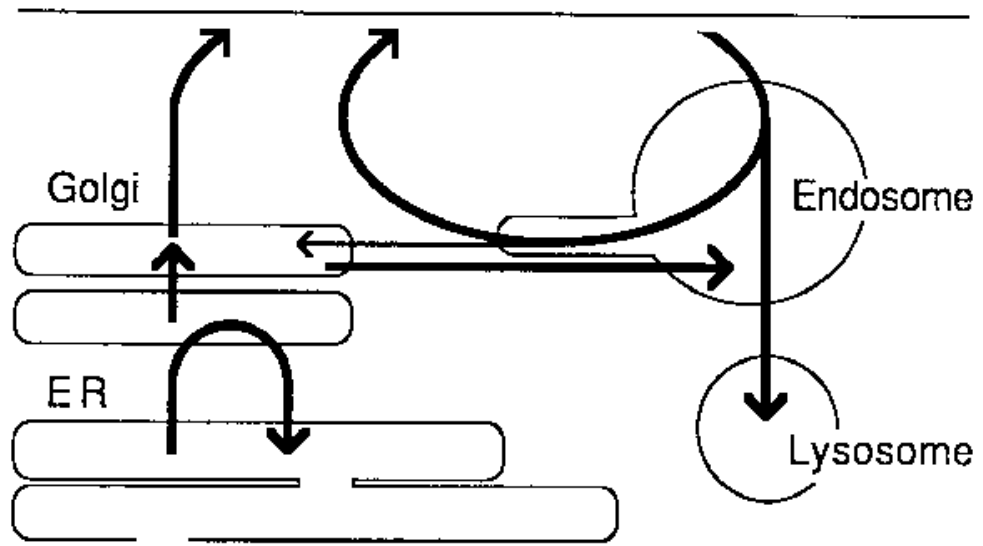

Figure 1 Pathways of vesicular traffic. Vesicles cycle between the starting point, the ER, and the cis Golgi (Pelham, this volume). Whether or not the intra-Golgi transport contains a unidirectional step is unclear at present. At the trans end of the Golgi the vesicle components are delivered to the plasmalemma and from it into the endocytic pathway. A shortcut connects the trans Golgi network via a prelysosomal compartment to the lysosomes; the latter present a point of no return for vesicular traffic (Kornfeld \& Mellman, this volume).

cholesterol (Orci et al 1981). The composition of endocytic coated vesicles and endosomal membranes is similar to that of the plasma membrane. In virtually all cases studied, they contained high levels of cholesterol, SPH, and PS (Dickson et al 1983; Luzio \& Stanley 1983; Evans \& Hardison

Table 1 Typical lipid composition of some intracellular organelles of rat liver

\section{Mitochondrial membrane}

Phospholipids:

$\begin{array}{rrrr}0.5 & 2.5 & 16.0 & 20.3 \\ 40.3 & 58.4 & 39.3 & 39.7 \\ 4.6 & 10.1 & 7.7 & 4.5 \\ 0.7 & 2.9 & 9.0 & 1.7 \\ 34.6 & 21.8 & 23.3 & 14.1 \\ 17.8 & 1.1 & 1.0 & 1.0 \\ 0.2^{\mathrm{a}} & & & 7.0 \\ & & & \\ 0.03 & 0.08 & 0.40 / 0.76^{\mathrm{b}} & 0.49^{\mathrm{b}}\end{array}$

${ }^{\text {a }}$ Data from Wherrett \& Huterer 1972.

${ }^{b}$ Data from Colbeau et al 1971.

Data expressed as \% of total phospholipid phosphorus (from Zambrano et al 1975, with permission). 
1985; Helmy et al 1986; Belcher et al 1987; Urade et al 1988). ${ }^{3}$ Lysosomal membranes have a plasma membrane-like composition, with high levels of cholesterol and SPH (Table 1) and GSLs (Henning \& Stoffel 1973). However, the level of PS is very low. Moreover, the lysosome is the only organelle containing lysobisphosphatidic acid (LBPA; see Brotherus \& Renkonen 1977), a phospholipid with a remarkable structure.

\section{Lipid Composition of Organelles Not Connected by Vesicular Traffic}

Mitochondria have a characteristically simple lipid composition as they are devoid of GSL, and the levels of cholesterol, SPH, and PS are very low (Table 1). The mitochondrial inner membrane mainly consists of PC and $\mathrm{PE}$, and is unique in that it contains significant levels of cardiolipin (CL). The actual surface area covered by $C L$ is twice its mol percent: each CL molecule contains two diacylglycerophosphate moieties, while the other phospholipids have one. The mitochondrial PI is localized primarily in the outer membrane, which has PC and PE as its major lipids (see Daum 1985).

PC and PE also constitute the major phospholipids of the peroxisomal membrane ( 73 and 18\%, Crane \& Masters 1986), the remainder being PI and PS. As in mitochondria, no SPH is found. In contrast, some cholesterol seems to be present (Fujiki et al 1982), which is easily explained by the fact that it can be synthesized by peroxisomes (Appelkvist 1987; et al 1987).

\section{Quantitative Distribution of Lipids between the Various Organelles}

The enrichment of a lipid in a certain organelle does not imply that this organelle contains the bulk of that lipid as the relative membrane areas of different organelles have to be considered. The subcellular distribution of a lipid can be determined from the products of the concentration of the lipid in each organelle (mol/area) and the surface area of the organelle. When the lipid compositions of the organelles in BHK cells (Brotherus \& Renkonen 1977) are combined with the data on surface area (G. Griffiths,

\footnotetext{
${ }^{3}$ Interpretation of the differences between endosomal and plasma membrane fatty acyl compositions reported in the latter paper is difficult. The plasma membrane phospholipid composition was not plasma membrane-like, but looked like ER.
} 
R. Back, M. Marsh, submitted for publication), the following numbers are obtained: $90 \%$ of the LBPA is found in the lysosomes, and $90 \%$ of the cellular CL is assigned to the inner mitochondrial membrane. While this probably reflects a virtually complete confinement of LBPA and CL to specific organelles, none of the major lipids displays such a biased distribution. Of the typical plasma membrane lipids SPH and PS, only 30 $35 \%$ is present in the plasma membrane vs $11 \%$ of the total cellular PC and PE. Also the fraction of cellular cholesterol in the plasma membrane is remarkably low, 25-40\%. Similarly, GSLs are predominantly intracellular (Tanaka \& Leduc 1956; Weinstein et al 1970; Keenan et al 1972; Hansson et al 1986; Symington et al 1987). These numbers (cf van Meer 1987) are consistent with the similarity between the lipid compositions of trans Golgi, endosomes and lysosomes and that of the plasma membrane (see above), and with the finding that the total surface area of the former three compartments is of the same order as that of the plasma membrane (G. Griffiths, R. Back, M. Marsh, submitted for publication). The persistent idea that practically all GSL (Thompson \& Tillack 1985) and $90 \%$ of both SPH and cholesterol (Lange et al 1989) are located in the plasma membrane most likely results from the fact that in the experiments essentially no attempt was made to distinguish the contribution of the endosomal membranes and the trans Golgi from that of the plasma membrane.

\section{Transmembrane Lipid Asymmetry}

PLASMA MEMBRANE LIPID ASYMMETRY Evidence is accumulating to suggest that plasma membranes in most cells are organized like the well-characterized erythrocyte membrane (reviewed in Op den Kamp 1979; Zachowski \& Devaux 1989). Most of the choline-phospholipids SPH and PC are situated in the exoplasmic bilayer leaflet and most of the aminophospholipids PS and PE are on the cytoplasmic surface. SPH appears to be located in the exoplasmic leaflet exclusively (Allan \& Walklin 1988). Also PS is essentially unilateral, whereas significant fractions of PC and PE are present in both bilayer leaflets. GSLs are exclusively exoplasmic, while the scarce data on the distribution of cholesterol are contradictory (Zachowski \& Devaux 1989). Arguments will be provided in favor of a preferential location of cholesterol in the exoplasmic leaflet.

AN AMINO-PHOSPHOLIPID TRANSLOCATOR IN THE PLASMA MEMBRANE Eukaryotic plasma membranes are stable bilayers, with low rates of transbilayer mobility (flip-flop). GSLs do not translocate at all and this appears also to be the case for SPH (Zachowski \& Devaux 1989, see below). In the case of PC, two pools exist in the plasma membrane that intermix only very slowly due to $t_{1 / 2} \mathrm{~s}$ of flip-flop in the order of $10 \mathrm{~h}$ or 
more at $37^{\circ} \mathrm{C}$ (Pagano \& Sleight 1985; Zachowski \& Devaux 1989). ${ }^{4}$ Conversely, the amino-phospholipids appear to be actively translocated to the cytoplasmic leaflet by an energy requiring amino-phospholipid translocator (see Devaux 1988). Also spontaneous flip-flop of the aminophospholipids appears to be much faster than that of PC (see Pagano \& Sleight 1985; Zachowski \& Devaux 1989). Therefore the plasma membrane phospholipid asymmetry represents an energy consuming equilibrium state, possessing all necessary properties to be regulated. It has been suggested that since cytoskeletal elements possess the capability to interact with PS, they might stabilize the lipid asymmetry. It is, however, not obvious how such a skeleton would quantitatively trap PS in the cytoplasmic leaflet (see Zachowski \& Devaux 1989).

TRANS GOLGI AND ENDOCYTIC MEMBRANES ARE PLASMA MEMBRANE-LIKE Lipid asymmetry and a low flip-flop rate for PC are also characteristic for endocytic membranes (Sandra \& Pagano 1978). Moreover, fluorescent analogs of PC and SPH did not translocate to the cytoplasmic surface when going through the endocytic pathway (Pagano \& Sleight 1985; Koval \& Pagano 1989). Finally, fluorescent sphingolipids did not flip-flop from their luminal location in the Golgi complex (Lipsky \& Pagano 1985; van Meer et al 1987). In combination with the plasma membrane data, this suggests that membranes in the endocytic pathway (Figure 1) possess one exoplasmic pool of sphingolipids, two nonequilibrating pools of PC, and two pools of PE and of PS that readily equilibrate (at least at the plasma membrane).

RAPID PC FLIP-FLOP IN ER Studies on the lipid organization in the ER membrane have been prone to methodological artifacts (van Meer 1986). Still it has been reproducibly shown that PC, the major ER lipid, rapidly translocates across the ER membrane, possibly facilitated by a PC-specific pore, a "PC-flippase" (references in Bishop \& Bell 1988). This explains how the unilateral lipid synthesis on the cytoplasmic ER surface (Bell et al 1981) can yield a lipid bilayer. Synthesis of PE, PS, and PI at the ER also occurs on the cytoplasmic surface. Simple absence of flip-flop for these phospholipids would by itself create phospholipid asymmetry, with PC being the only luminal phospholipid. However, the transmembrane organization and flip-flop rates of PE, PS, and PI in the ER are unknown. PC flip-flop must be abolished along the exocytic route. Although the

${ }^{4}$ Flip-flop rates with a $t_{1 / 2}$ of about $30 \mathrm{~min}$ have been reported for SPH and PC in the plasma membrane of guinea pig cells. It is not obvious why the rate of flip-flop should be so "very species-dependent" (Sune et al 1988). 
mechanism remains unclear, the simplest solution would be retention of the putative PC-flippase in the ER.

\section{LIPID TRAFFIC AND SORTING}

\section{Generation and Maintenance of the Heterogeneous Distribution of Sphingolipids}

SPHINGOLIPIDS ARE SYNTHESIZED ON THE LUMINAL ASPECT OF A GOLGI MEMBRANE Any single cell expresses a limited number of GSLs, mostly one or two series each derived from ceramide by the stepwise addition of monosaccharides. Apart from ceramide synthesis in the ER, the various steps in GSL assembly occur on the luminal surface of subsequent cisternae of the Golgi complex (see Miller-Prodraza \& Fishman 1984; Lipsky \& Pagano 1985; Klein et al 1988). The implications of glycosyl-transferase activities at the cell surface (Pierce et al 1980) for GSL biosynthesis are unclear.

SPH is assembled by the energy independent transfer of phosphorylcholine from a PC molecule onto ceramide. In the early studies (e.g. Marggraf et al 1981; Voelker \& Kennedy 1982) the plasma membrane was proposed to be a major site of SPH biosynthesis. However during biosynthetic labeling of SPH with radioactive choline, the specific activity of plasma membrane SPH notably lagged behind that of SPH in a Golgi/ER fraction (Cook et al 1988). The synthesis of a fluorescent SPH has now been shown to occur on the luminal surface of the Golgi (see Pagano 1988), and not on the plasma membrane of interphase (Lipsky \& Pagano 1985; G. van Meer, submitted for publication) and mitotic cells (Kobayashi \& Pagano 1989); an alternative explanation for the enrichment of SPH in the plasma membrane.will be provided.

GSLs and SPH apparently do not flip-flop. Their exclusive location in the exoplasmic leaflets of the various organelles must, therefore, be established during biosynthesis. This also explains why GSLs and SPH are exclusively found in organelles connected by vesicular transport (Figure 1$)^{5}$

SPHINGOLIPID SORTING ALONG THE EXOCYTIC PATHWAY IN EPITHELIAL CELLS A better understanding of the intracellular transport and sorting of sphingolipids has been obtained from studying their enrichment in a domain of the plasma membrane of epithelial cells.

\footnotetext{
${ }^{5}$ Various proteins that in vitro displayed GSL transfer activity have now been identified as activator proteins for GSL degradation in the lysosomal lumen (see Fürst et al 1988; O'Brien et al 1988). The physiologic function of a putative "SPH specific exchange protein" (Dyatlovitskaya et al 1982) is unclear.
} 
The plasma membrane of epithelial cells is divided into an apical and a basolateral domain by tight junctions that encircle the apex of each cell. The apical domain, which a number of criteria indicate to be a specialized part of the plasma membrane (Simons \& Fuller 1985), is enriched in GSLs, at the expense of $\mathrm{PC}$, when compared to the basolateral plasma membrane. An apical enrichment of SPH in some kidney cells has been reported (see Venien \& Le Grimellec 1988), but unfortunately not the GSL content of the corresponding membranes (see Simons \& van Meer 1988; van Meer 1988). Where studied, the levels of GSLs plus SPH are high enough to completely cover the apical membrane, which implies that the glycerophospholipids must be confined to the cytoplasmic bilayer leaflet. Indeed experimental evidence indicates that only a minor fraction of glycerophospholipids is accessible to phospholipases, exchange proteins, and the chemical reagent trinitrobenzene sulfonate (Barsukov et al 1986; Venien \& Le Grimellec 1988).

While current evidence indicates that some membrane proteins display a polarized distribution even in the absence of tight junctions (e.g. Salas et al 1988), the intactness of the latter is required for maintaining the compositional difference between the two plasma membrane domains in the case of lipids. The tight junctions block lipid diffusion. The barrier is exclusive to the exoplasmic leaflet of the bilayer (references in Simons \& van Meer 1988). Therefore the lipid differences between the two domains reside in that outer leaflet. The cytoplasmic leaflets of the two domains share the same composition, thus lipids can freely diffuse between them. The transbilayer distribution of the various phospholipids in the basolateral membrane can now be calculated from its total phospholipid composition and that of its cytoplasmic leaflet (which is identical to that of the total apical membrane). In this way $75-90 \%$ of the PC was assigned to the exoplasmic leaflet vs $10-35 \%$ of the PE (van Meer \& Simons 1986). In addition, an endogenously synthesized fluorescent SPH was exclusively localized to the exoplasmic leaflet (van Meer et al 1987). The overall phospholipid asymmetry in the basolateral plasma membrane domain of epithelial cells is thus strikingly similar to that in the erythrocyte membrane.

Lipid polarity in epithelia is generated by lipid sorting The biosynthetic transport of apical and basolateral lipids has been studied using endogenously synthesized fluorescent analogs of glucosylceramide, a typical apical lipid, and SPH, which is not polarized in the cells used (van Meer et al 1987; G. van Meer, submitted for publication). Due to the fatty acid on their backbone, the analogs, N-6[7-nitro-2,1,3-benzoxadiazol-4-yl] aminocaproyl sphingosine-, are readily exchangeable between membranes, in contrast to the natural lipids. The appearance of the analogs on the 
outside of the plasma membrane could then be assayed by "backexchange", specific extraction of the fluorescent lipid from the surface by unlabeled liposomes or BSA (Lipsky \& Pagano 1985). Moreover, confinement of the fluorescent lipids to discrete intracellular structures, in this type of experiment, is most easily explained by luminal localization. The conclusions from our studies were $(a)$ fluorescent glucosylceramide and SPH were synthesized on the luminal, exoplasmic leaflet of an intracellular organelle membrane, and this most likely was a Golgi membrane; $(b)$ the lipids did not travel to the ER; (c) $90 \%$ of both lipids was transported to the cell surface within $2 \mathrm{~h}$ at $37^{\circ} \mathrm{C}$, with a $t_{1 / 2}$ of $20-30 \mathrm{~min} ;(d)$ sidedness was completely maintained, which indicated that transport was mediated by carrier vesicles: the fluorescent lipids ended up exclusively in the exoplasmic leaflet of the plasma membrane; $(e)$ two to threefold more glucosylceramide was delivered to the apical than to the basolateral surface vs equal amounts of the SPH analog. Because the experiments were set up such that redistribution by endocytosis could be excluded, lipid sorting had occurred prior to delivery to the cell surface. In analogy to the conclusion that apical plasma membrane proteins are sorted from basolateral and lysosomal proteins in the trans Golgi network (Simons \& Fuller 1985; Matlin 1986; Griffiths \& Simons 1986; Geuze et al 1987), we concluded that the trans Golgi network is also the site of exocytic lipid sorting (Simons \& van Meer 1988).

Lipid sorting by GSL microdomain formation To generate lipid polarity in epithelial cells, apical exoplasmic GSLs must be separated from PC, which is enriched in the exoplasmic leaflet of the basolateral surface. The evidence listed above justifies the conclusion that newly synthesized GSLs and SPH are transported from the trans Golgi to the plasma membrane by carrier vesicles. Arguments will be presented below indicating that this must also be the case for newly synthesized exoplasmic PC. As a consequence, the lipid sorting step in the exocytic pathway will necessarily involve the lateral segregation of GSLs from PC in the luminal bilayer leaflet of the trans Golgi network. Immunocytochemically a lateral segregation has been visualized in this organelle for membrane proteins with different intracellular destinations (Geuze et al 1987). Interestingly, GSLs differ from PC in that they possess the capacity to self-associate by hydrogen bonding. The available data fit a model (Simons \& van Meer 1988) where apical proteins and GSLs form a microdomain on the luminal surface of the trans Golgi membrane at the site of apical vesicle budding. The interaction between the proteins and GSLs may be direct, or mediated by a putative sorting protein. In some way such a sorting protein would also be involved in defining the apical destination of the resulting vesicle, for which interactions with proteins on the cytoplasmic surface would seem 
necessary (for discussions see Burgess \& Kelly 1987; Pelham, Kornfeld \& Mellman, Hurtley \& Helenius, this volume). Few data are available on the type of bulk interactions between proteins and GSLs that would be required in such a model (Devaux \& Seigneuret 1985). Still, the self-association properties of GSLs and their omnipresence in epithelia make them attractive candidates for being instrumental in the formation of microdomains. It is tempting to speculate that they are essential for generating the specialized apical plasma membrane domain. In addition, the same sorting mechanism might play a role in the polarized exocytic transport in a number of other polarized but nonepithelial cells like cytotoxic T cells, neurons, and osteoclasts.

SPHINGOLIPID SORTING IN CIS GOLGI The conclusion that (fluorescent) sphingolipids are not transported from their site of synthesis in the Golgi back into the ER explains the low concentration of sphingolipids in the ER. Recent evidence suggests that vesicular transport between the cis Golgi and the ER is bidirectional and that resident ER proteins that have entered cis Golgi are sorted into the return pathway (Pelham, this volume). Therefore, if GSLs and SPH are present in cis Golgi, which is unclear at present, they must be excluded from that return traffic by lipid sorting. In the case that sphingolipid biosynthesis would be localized in a more distal cisterna, the question whether lipid sorting occurs depends on the nature of the transport connection between cis Golgi and that cisterna. Various alternatives for SPH (and GSL) transport and sorting are presented in Figure 2. An alternative mechanism that would result in unidirectional transport of SPH has been proposed by Wieland et al (1987; cf Pagano

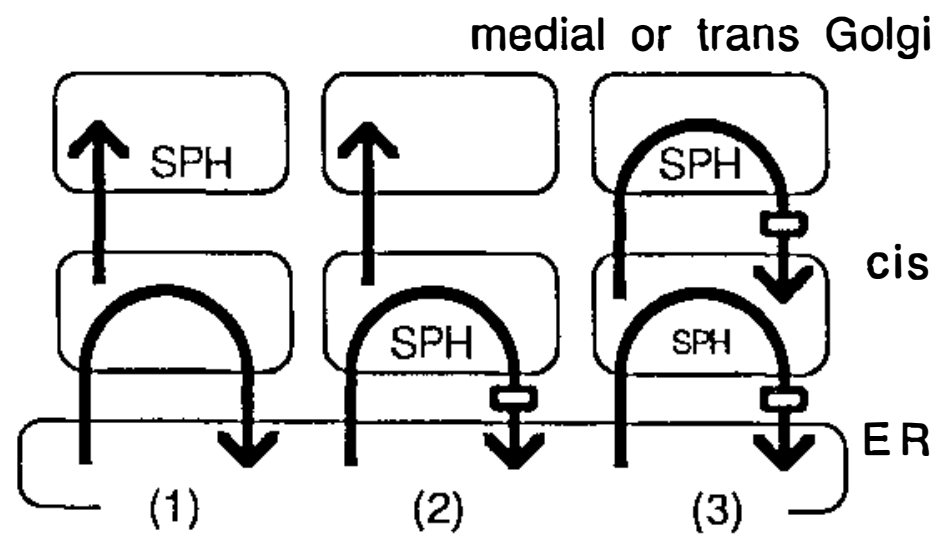

Figure 2 SPH does not reach ER. Possible (alternative) explanations: (1) SPH synthesis past a unidirectional vesicular transport step: no SPH sorting. (2) SPH synthesis bef ore a unidirectional step: SPH excluded from return traffic. (3) Bidirectional vesicular traffic throughout the Golgi stack: multiple SPH sorting steps. Arrows: vesicular traffic, blocks: exclusion of SPH. 
1988). Whereas import of material into cis Golgi is vesicular, return traffic might occur by lipid monomers. Although the data of Pelham \& coworkers (this volume) make this alternative unlikely, the model has interesting consequences: traffic of retained components, proteins, $\mathrm{SPH}$, and possibly also cholesterol, would be vectorial and they would be concentrated at once. Although the alternatives without sorting provide the easy explanation for the cellular distribution of sphingolipids, the sorting variants (2) and (3) (Figure 2) have attractive implications for the mechanism by which cholesterol is concentrated on its way to the plasma membrane (see below). The action of the subsequent sorting steps in option (3) might result in efficient sorting even if each individual sorting event, like epithelial sphingolipid sorting, were rather leaky.

Sorting, in this case, involves the exclusion of the (luminal) sphingolipids from a budding return vesicle. If phospholipid asymmetry is established in the ER by the presence of a PC-specific flippase, as discussed above, the luminal leaflet of the ER (and cis Golgi) membrane contains PC. In that case the Golgi sorting step segregates the sphingolipids into the exocytic vesicles and PC into the return vesicles. If asymmetry is established only later in the Golgi stack, the sphingolipids on the luminal surface would have to be sorted from a mixture of phospholipids. One may speculate that sphingolipid sorting in the Golgi stack is the more general case of the sphingolipid sorting in the trans Golgi of epithelial cells. Whereas in the stack, SPH would participate in hydrogen bonding with the GSLs and both GSLs and SPH would be separated from glycerophospholipids, in the trans Golgi network of epithelial cells, GSLs are sorted from PC whereby incorporation of SPH in the GSL microdomain seems to depend on the specific conditions in the various cell types.

UNIDIRECTIONAL VESICULAR TRANSPORT IN THE TRANS GOLGI? After synthesis in the Golgi, GSLs and SPH are transported to the plasma membrane with $t_{1 / 2} \mathrm{~S}$ of $20-30 \mathrm{~min}$, a process inhibited at $15^{\circ} \mathrm{C}$ (Miller-Prodraza \& Fishman 1984; Lipsky \& Pagano 1985; van Meer et al 1987). From the plasmalemma they can enter the endocytic pathway (Figure 1). Although the bulk of the lipids recycles from the endosomes straight to the plasmalemma, it has been demonstrated that a small fraction of the GSLs recycles to the plasma membrane via the Golgi (Fishman et al 1983; Klein et al 1988). It appears that, as with recycling membrane proteins (see Neefjes et al 1988), recycling GSLs may be limited to the trans Golgi cisternae and hence excluded from the proximal cisternae of the Golgi. After implantation into the plasma membrane, simple gangliosides were unable to reach the Golgi site where they could be converted to a more complex form (see Klein et al 1988). As argued above such exclusion could 
be the result of sphingolipid sorting or, alternatively, of a unidirectional transport step of GSLs in the trans Golgi. Although unidirectional transport in the exocytic pathway has been suggested before (Cohen \& Phillips 1980), and the advantages of its location in the trans Golgi discussed (Rothman 1981), there is at present no direct evidence against vesicles returning from the trans Golgi into the Golgi stack. ${ }^{6}$

VESICULAR SPHINGOLIPID TRANSPORT IN THE ENDOCYTIC PATHWAY Pagano \& coworkers have demonstrated (see Pagano \& Sleight 1985; Koval \& Pagano 1989) that fluorescent analogs of PC and SPH, after insertion into the exoplasmic leaflet of the plasma membrane of fibroblasts, are endocytosed into early peripheral endosomes and subsequently transported to perinuclear endosomes. As argued above, from the types of probes used it could be concluded that the transport was vesicular and that no translocation of the PC and SPH had occurred to the cytoplasmic bilayer leaflet in any endocytic organelle. The $t_{1 / 2}$ for a complete round of SPH recycling was estimated to be about $40 \mathrm{~min}$ (Koval \& Pagano 1989).

SPHINGOLIPID SORTING IN THE ENDOSOMES Since a vesicular pathway connects the endosomes to the lysosomes (Figure 1) there must be a continuous flux of lipids into the latter. For lipids in the exoplasmic leaflet this flux appears not to be random. Fluorescent analogs of SPH present in the luminal leaflet are preferentially recycled to the plasma membrane (Koval \& Pagano 1989). Cellular gangliosides have half-lives in the order of $10-50 \mathrm{~h}$ and therefore do not seem to reach the lysosomes efficiently either (see Miller-Prodraza \& Fishman 1984). In contrast, N-Rh-PE, a fluorescent PE, was quantitatively shuttled to the lysosomes within $1 \mathrm{~h}$ when present in the exoplasmic leaflet of the plasma membrane (J. Davoust \& M. Kail, paper in preparation; J. W. Kok, M. Ter Beest, G. Scherphof, $\&$ D. Hoekstra, paper in preparation). Lipids destined for the lysosomes can thus be sorted, like proteins, from recycling lipids in the endosomes (see Geuze et al 1987). Additional lipid sorting at the level of the coated pits of the plasma membrane may occur. Little is known about the mechanism of lipid sorting to the lysosomes; however the process apparently occurs in the luminal leaflet of the endosomal membrane and must involve lateral segregation of lipids.

For lipids the vesicular endocytic route to trans Golgi appears to be quantitatively of far less importance than direct recycling from the endosomes to the plasmalemma (Fishman et al 1983; Klein et al 1988; Koval

\footnotetext{
${ }^{6}$ In a few cases, access of cell surface markers to cisternae in the Golgi stack has been reported (see Farquhar 1985). The interpretation of these observations is unclear at present (cf Griffiths \& Simons 1986; Neefjes et al 1988).
} 
\& Pagano 1989). Nothing is known concerning possible lipid sorting events along this pathway.

Preliminary experiments on transcytosis, the vesicular connection between the apical and basolateral plasma membrane domains in epithelial cells, have demonstrated that this pathway involves sphingolipid sorting similar to that in the exocytic pathway in these cells and have suggested the endosomes as the site of sorting (G. van Meer, unpublished observations).

\section{Generation and Maintenance of the Heterogeneous Distribution of Cholesterol ${ }^{7}$}

CHOLESTEROL BIOSYNTHESIS IN THE ER According to the general view of the past 20 years, cholesterol biosynthesis occurs on the ER (Reinhart et al 1987). In addition, a (minor) independent pathway has been shown to exist in the peroxisomal lumen (Appelkvist 1987; Thompson et al 1987). Lange \& colleagues have presented cell fractionation data from which they proposed that subsequent steps in the biosynthetic process were topographically heterogeneous. It is unclear, however, whether the various peaks on their sucrose gradients represent contiguous regions of a compound structure (the ER) or entirely discrete, perhaps hitherto undescribed, organelles (Reinhart et al 1987; Lange \& Muraski 1988).

VESICULAR CHOLESTEROL TRANSPORT TO THE PLASMA MEMBRANE All evidence agrees that the transport of newly synthesized cholesterol from its site of synthesis to the plasma membrane is vesicular: it is inhibited at $15^{\circ} \mathrm{C}$, and influenced by energy inhibitors (Lange \& Matthies 1984; Kaplan \& Simoni 1985b; Slotte et al 1987). Probably due to methodological problems, however, the interpretation of the transport data is still controversial. On the one hand, the cholesterol transport appeared to be vectorial with a short (10 min; DeGrella \& Simoni 1982) or long $t_{1 / 2}$ (1-1.5 h; Lange \& Matthies 1984). On the other hand, Kaplan \& Simoni (1985b) have concluded that the vesicular transport represents two-directional equilibration

\footnotetext{
${ }^{7}$ Compared to the methods used to localize other cellular lipids, those for cholesterol suffer from severe drawbacks. Spontaneous cholesterol exchange (see Phillips et al 1987) has a $t_{1 / 2}$ on the order of $2 \mathrm{~h}$ at $37^{\circ} \mathrm{C}$, which is slow as compared to cellular transport processes. Since all cholesterol in the endocytic compartments of Figure 1 will also take part in the exchange process, the method results in an overestimate of plasma membrane cholesterol. A second method involves the use of cholesterol oxidase. The results depend on how the method is used (see Brasaemle et al 1988). It appears that more than just the plasma membrane cholesterol is accessible to externally added oxidase. The observation that newly synthesized cholesterol is not available to the enzyme (see Lange \& Muraski 1988) seems insufficient as a control. Proof has to be provided that endosomal cholesterol is not oxidized. Also the interpretation of results obtained with filipin has led to serious problems (Yeagle 1985). Finally, the limitations of cell fractionation are discussed above.
} 
of ER cholesterol with that in the plasma membrane with a $t_{1 / 2}$ of about 10 min. Other observations make this highly unlikely. Equilibration of exogenously inserted plasma membrane cholesterol with that in the ER, assayed as access of the cholesterol to the site of esterification, was shown to have a $t_{1 / 2}$ in the order of 2-5 days (see Lange \& Matthies 1984; Slotte \& Bierman 1987).

SORTING OF CHOLESTEROL INTO THE EXOCYTIC PATHWAY The cholesterol concentration in the plasma membrane is much higher than that in the ER (Table 1). Therefore compared to the bulk membrane lipids, the phospholipids, cholesterol must have been concentrated along the pathway. The putative SPH sorting in the Golgi suggests a mechanism for this. A preferential interaction of cholesterol with SPH in comparison with other phospholipids (the weakest interaction being with PE) has been observed in numerous studies (Demel et al 1977; Gardam et al 1989; reviewed in Barenholz \& Thompson 1980; Phillips et al 1987). With the estimation by Wieland et al (1987) that about $50 \%$ of the ER lipid cycles through the cis Golgi every $10 \mathrm{~min}$, and with SPH present in the cis Golgi, optimal contact of this SPH with the ER cholesterol would be guaranteed. Cholesterol may be incorporated into an SPH microdomain and subsequently be sorted as a complex to the medial Golgi and further out. In fact, the apparent cholesterol concentration gradient over the stack, measured by the density of filipin-cholesterol complexes (Orci et al 1981), would be most consistent with the model for SPH sorting effected at a series of steps, Figure 2 (3).

Some eukaryotes, like Acanthamoeba, do not contain SPH, but do enrich sterols in the plasma membrane (Dawidowicz 1987), probably during vesicle transport (Mills et al 1984). It is of possible evolutionary interest that cholesterol also displays a preference for disaturated PC, close to that for SPH (Lange et al 1979; see Phillips et al 1987): the mechanism of cis Golgi sorting may originally have used disaturated phospholipids. These may still be required in the sorting as there is too little SPH to complex cholesterol on a one to one basis. Plasma membranes are enriched in disaturated phospholipids (e.g. Keenan \& Morré 1970; Colbeau et al 1971).

As a consequence of a preferential cholesterol-SPH interaction, ${ }^{8}$ the

\footnotetext{
${ }^{8}$ The importance of SPH for maintaining the heterogeneous distribution of intracellular cholesterol is illustrated by the fact that hydrolysis of plasma membrane SPH resulted in a shif $t$ of cholesterol from the plasma membrane to intracellular membranes, while a shift out of the ER was observed af ter cellular uptake of exogenous SPH: the latterinduced a number of effects known to be mediated by lowering the ER cholesterol concentration (see Slotte \& Bierman 1988). Finally, it is interesting to note that the biosynthesis of all sphingolipids appears to be controlled by low density lipoprotein (cholesterol) uptake (Chatter jee et al 1986).
} 
bulk of the cholesterol would be situated in the luminal, exoplasmic bilayer leaflet of membranes in the Golgi and the endocytic compartments (Figure 1)! Although $\mathrm{X}$-ray studies have indeed shown this to be the case in myelin, the plasma membrane of Schwann cells and oligodendrocytes (Caspar \& Kirschner 1971), in general the sparse data available on the transmembrane distribution of cholesterol in cellular membranes are contradictory, even for the well-characterized erythrocyte (Zachowski \& Devaux 1989).

MONOMERIC CHOLESTEROL DIFFUSION Cholesterol equilibration between membranes by spontaneous exchange with a $t_{1 / 2}$ of about $2 \mathrm{~h}$ is slow compared to vesicular traffic (Phillips et al 1987). This value was obtained in vitro and may have been dependent on membrane collisions (Steck et al 1988). Such collisions may be limited in the cell where the process may occur more slowly. Still nonvesicular transport of cholesterol seems necessary in at least two cases (and will therefore be a common cytoplasmic process). ${ }^{9}$ One is transport of cholesterol to the inner mitochondrial membrane of steroidogenic cells for the first step of the hormone production, the side chain cleavage, the second is the use of cholesterol derived from low density lipoproteins in the lysosomes for cholesterol ester synthesis in the ER.

CHOLESTEROL TRANSPORT INTO THE MITOCHONDRION During steroidogenesis large amounts of cholesterol are transported from lipid droplets to the inner mitochondrial membrane for the initial step of hormone production, the side chain cleavage. A large body of evidence suggests that a 13.5 -kd protein, sterol carrier protein 2 (SCP-2), has a crucial role at two steps of the transport process: the cytoplasmic transport to the outer mitochondrial membrane and the transfer from the outer to the inner membrane (see Scallen et al 1985). SCP-2, which has independently been characterized as possessing a nonspecific lipid transfer activity in vitro, also stimulates a number of metabolic conversions of cholesterol. For the time being, mechanistic interpretations concerning the cytoplasmic actions of this complicated protein SCP-2 (van Amerongen et al 1989, and references therein) are frustrated by immunocytochemical studies that have

\footnotetext{
${ }^{9}$ The heterogeneous distribution of cholesterol could be a simple consequence of cytoplasmic exchange in combination with different affinities of the various intracellular membranes for cholesterol. Such differences were indeed observed between microsomal, mitochondrial, and plasma membrane fractions (Wattenberg \& Silbert 1983), but not to an extent that could explain the level of cholesterol enrichment in the plasma membrane as compared to, for example, the ER. However, interpretation of the data was complicated by the fact that the experimental setup was based on the premise that an exchange equilibrium would exist between the various cholesterol pools. As described above, such an equilibrium appears not to apply to the cholesterol in the exoplasmic leaflet of the plasmalemma and that in the ER.
} 
localized the protein to the peroxisomal matrix (Tsuneoka et al 1988; van Amerongen et al 1989) instead of to the cytosol (see Scallen et al 1985). In fact, this rather suggests a role for SCP-2 in peroxisomal cholesterol synthesis.

Transport of cholesterol from the lipid droplet to the site of side chain cleavage on the inner aspect of the inner membrane requires at least four discrete steps: cytoplasmic transport to the outer membrane, translocation across the outer membrane bilayer, transfer across the intermembrane space to the inner membrane (with the possible involvement of the "contact sites"), and translocation across the inner membrane. Although various inhibitors and activators of the overall transport have been characterized, the actual mechanism of each step remains obscure (reviewed in Lambeth et al 1987; van Amerongen et al 1989).

CHOLESTEROL FLIP-FLOP IN LYSOSOMES BUT NOT PLASMA MEMBRANE? Cholesterol derived from low density lipoprotein starts to exert effects at the level of the ER, i.e. stimulation of cholesterol ester synthesis and downregulation of cholesterol synthesis after just $3 \mathrm{~h}$ (Liscum \& Faust 1987). These regulatory functions appear to depend on the actual insertion of the cholesterol into the ER membrane (Orci et al 1984; Davis \& Poznansky 1987). Similar effects could be obtained by raising the plasma membrane cholesterol concentration by transfer from cholesterol-rich liposomes, but only after $24 \mathrm{~h}$ (Robertson \& Poznansky 1985). The simplest explanation for the fact that lysosomal cholesterol does and exoplasmic plasmalemma cholesterol does not participate in cytoplasmic transport processes would be that cholesterol flip-flops to the cytoplasmic leaflet of the lysosomal membrane but not of the plasma membrane. ${ }^{10}$ Unfortunately, the rate of cholesterol flip-flop is also a matter of controversy (see Zachowski \& Devaux 1989).

In summary, in cells rapid transport of cholesterol occurs by means of carrier vesicles $\left(t_{1 / 2}\right.$ of about $\left.30 \mathrm{~min}\right)$, while cholesterol also redistributes by monomeric exchange ( $t_{1 / 2}$ probably longer than $2 \mathrm{~h}$ ). The heterogeneity in cholesterol content in vivo is best explained by cholesterol sorting in the Golgi complex (based on a differential affinity for the various phospholipid classes) in combination with preferential localization of cholesterol in the

\footnotetext{
${ }^{10}$ Either flip-flop in the lysosomes or the aqueous transfer of cholesterol is inhibited in type C Niemann-Pick disease, while biosynthetic (vesicular) transport is not (Liscum et al 1989). The enormous increase in lysosomal cholesterol in this disease observed by many authors (Blanchette-Mackie et al 1988; Liscum et al 1989) was accompanied by a relatively modest rise of the cholesterol level in the trans Golgi (Blanchette-Mackie et al 1988). Interpretation of this observation in terms of transport is difficult. Possibly, the vesicular endosome-trans Golgi route (Figure 1) is involved.
} 
exoplasmic leaflet of the bilayer, and the absence of cholesterol flip-flop in Golgi membranes, plasmalemma, and endosomal membranes.

\section{Generation and Maintenance of the Heterogeneous Distribution of Glycerophospholipids}

PHOSPHATIDYLCHOLINE DISTRIBUTION AND TRANSPORT Cellular PC is synthesized in the ER and the Golgi by the CDP-choline pathway and to a small extent by the stepwise methylation of PE (Jelsema \& Morré 1978; Bell et al 1981; Vance \& Ridgway 1988), whereas straight incorporation of choline by a base exchange reaction appears to be a minor route (see Yaffe \& Kennedy 1983; Figure 3). The creation of the ER bilayer is made possible by the presence of a PC-pore or "flippase" (see Bishop \& Bell 1988; Devaux 1988), but somewhere in the Golgi stack this potential to translocate PC across the bilayer is lost: at least from the trans Golgi onwards throughout the endocytic pathway (Figure 1), transport has to account for two PC pools that do not rapidly intermix (see above). Kaplan \& Simoni (1985a) have described how newly synthesized PC rapidly equilibrated with that in the plasma membrane $\left[t_{1 / 2}\left(37^{\circ} \mathrm{C}\right) \ll 2 \mathrm{~min}\right]$. However, when looking for two-pool kinetics, it is evident from the data that a significant fraction of the newly synthesized PC reached the plasma membrane with a $t_{1 / 2}$ of $30-60 \mathrm{~min}$, a process that was influenced by energy poisons and inhibited at $15^{\circ} \mathrm{C}$, all of which are characteristics of vesicular transport. The obvious prediction that this PC fraction represents the exoplasmic $\mathrm{PC}$ pool will have to be proven by a direct experiment.

Transport of newly synthesized PC (and PE) by a special class of phos-

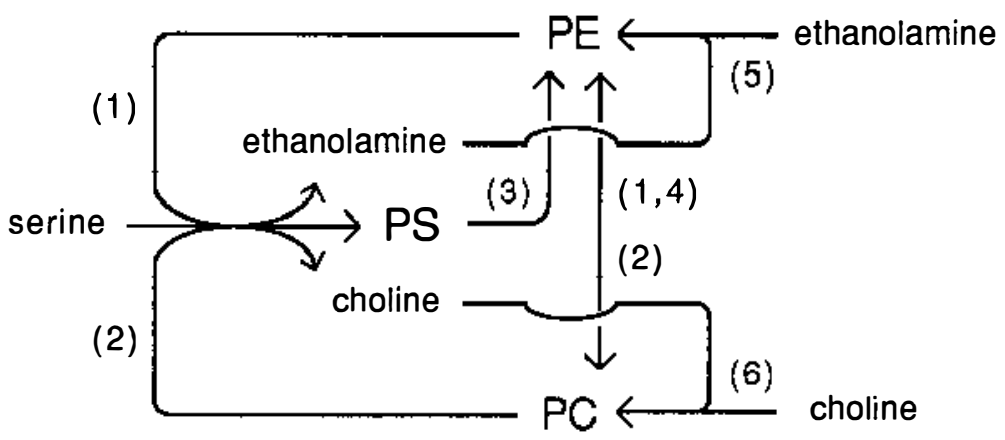

Figure 3 Import of head groups into the cellular phospholipid metabolism: (1) baseexchange from PE, (2) base-exchange from PC, (3) PS-decarboxylase, (4) PE methylation, (5) ethanolamine kinase; CTP-phosphoethanolamine cytidylyltransferase; CDP-ethanolamine: diacylglycerol ethanolamine phosphotransferase, (6) choline kinase; CTP-phosphocholine cytidylyltransferase; CDP-choline: diacylglycerol choline phosphotransferase. 
pholipid-rich vesicles has been reported to be responsible for the rapid plasma membrane growth during mitosis (Bluemink et al 1983) and cell aggregation of Dictyostelium (De Silva \& Siu 1981).

Newly synthesized PC equilibrated with the outer mitochondrial membrane PC pool with a $t_{1 / 2}$ in the order of $30 \mathrm{~min}$ (McMurray \& Dawson 1969; Blok et al 1971) or faster (Yaffe \& Kennedy 1983). ${ }^{11}$ Candidates for mediating this equilibration, which according to the present view is not vesicular, are two transfer proteins that possess the capability to transport PC monomers across an aqueous barrier; one has a strict specificity for PC, the other is specific for PI and PC (reviewed in Helmkamp 1986). ${ }^{12}$ The need for such rapid equilibration is unclear at present. However, it is clear that in order for mitochondria to grow a net transfer of PC into the mitochondria is required, since mitochondria do not synthesize PC themselves. Several investigators have demonstrated that the PC-specific transfer protein can yield such net transfer in vitro. However, since the experiments were performed under conditions where the PC concentrations of donor and acceptor membranes were far from equilibrium (discussed in Helmkamp 1986), these results cannot be simply extrapolated to the situation in the cell. How mitochondria, or for that matter peroxisomes, expand their surface, therefore remains an enigma.

Transfer of PC and PE between the outer and inner mitochondrial membranes is much slower than other intracellular PC traffic, with a $t_{1 / 2}$ in the order of 0.5-3 h (Bygrave 1969; McMurray \& Dawson 1969; Blok et al 1971; Eggens et al 1979). In contrast with the rapid PC flip-flop in the outer membrane, translocation of a spin-labeled PC across the inner membrane was found to be extremely slow (Rousselet et al 1976).

PHOSPHATIDYLINOSITOL TRANSPORT Essentially all PI is synthesized in the ER (Jelsema \& Morré 1978). It has been known for 20 years that PI equilibrates between ER and mitochondria within minutes (McMurray \& Dawson 1969), and a transfer protein with a specificity for PI and PC was later characterized. Inositol lipids, as intermediates in the polyphosphoinositol signal transducing system, display a high turnover in the cytoplasmic leaflet of the plasma membrane. Therefore replenishment of plasma membrane PI is required. Although vesicles traveling from the

\footnotetext{
${ }^{11}$ Equilibration of the total outer mitochondrial membrane PC pool within less than an hour predicts a relatively rapid PC flip-flop in this membrane, a property recently established by a direct in vitro experiment (K. Nicolay \& R. Hovius, paper in preparation).

${ }^{12}$ The PC and PI/PC specific transfer proteins may also be responsible for the rapid PC equilibration observed by Kaplan \& Simoni (1985a). However, as for sterol carrier protein 2 , the location in the cytosolic compartment remains to be demonstrated unequivocally for both transfer proteins.
} 
ER to the plasma membrane will undoubtedly carry PI, a more rapid mechanism of PI transfer has been proposed based on the dual specificity of the PI/PC transfer protein (see Helmkamp 1986; Van Paridon et al 1987). Since the PI level is lower in the plasmalemma than in the ER membrane, the PI/PC protein has a lower probability of picking up PI as compared to $\mathrm{PC}$ in the plasma membrane than in the ER; this could result in a net flux of PI towards the plasmalemma and a return flow of PC.

PHOSPHATIDYLSERINE DISTRIBUTION AND TRANSPORT PS is synthesized by PS synthase, an enzyme mediating base-exchange, primarily in the ER but also in the Golgi and the plasma membrane (Figure 3, Jelsema \& Morré 1978; Voelker 1985; Vance \& Vance 1988). Recent evidence points at PC as the major substrate of the reaction (see Kuge et al 1986; Miller \& Kent 1986; Voelker \& Frazier 1986). PS is enriched at least threefold in the plasma membrane (Table 1), and probably in all membranes of the endocytic pathway as compared to the other organelles (Luzio \& Stanley 1983; Urade et al 1988; cf Evans \& Hardison 1985). In the plasma membrane a specific enzyme maintains its location in the cytoplasmic leaflet (see Devaux 1988). ${ }^{13}$

Two considerations may be relevant for understanding how PS is concentrated in the cytoplasmic leaflet of the plasma membrane and the membranes of the endocytic pathway (Figure 1). (a) As PS, like other phospholipids, is subject to vesicular transport, traffic back into the Golgi should be prevented; this could imply PS sorting in the cytoplasmic leaflet of the Golgi by some new mechanism (cf SPH/cholesterol sorting in the exoplasmic leaflet). Interestingly, plasma membrane PS has been reported to have a much more saturated fatty acyl composition than PS in the ER (Keenan \& Morré 1970). (b) Significant monomeric exchange of PS occurs in the cytoplasm, but, as for cholesterol, the rate of this process appears to be much lower than that of vesicular transport (see below). As an alternative to PS sorting, the enhanced PS concentrations in the plasmalemma and the membranes of the endocytic pathway may be explained by these membranes having a higher affinity for PS. A reason for such an enhanced affinity of the former membranes as compared to the ER and

\footnotetext{
${ }^{13}$ The presence of the "amino-phospholipid translocator" and the fact that spontaneous flip-flop of PS across the plasma membrane is faster than that of the other phospholipids, suggest that PS is central to some regulatory mechanism. In line with the coupled bilayer hypothesis by Sheetz \& Singer (1974), the mechanism may control the relative surface areas and lateral pressure of each leaflet of the plasma membrane bilayer. One could even envisage how the ATP-requiring translocator could be the driving force behind endocytic vesicle budding from the flat plasma membrane. In this view, apart from sorting considerations, clathrin might be required to translate the pressure increase in the cytoplasmic leaflet into a local site of high curvature (see also Devaux 1988).
} 
the outer mitochondrial membrane may be the fact that they have a membrane potential that is positive on the exoplasmic side (e.g. Fuchs et al 1989): desorption of the negatively charged PS into the cytoplasm, which is against the electrical field, requires more energy than diffusion from the other organelles (independent of whether exchange is spontaneous or protein-mediated).

It has been proposed that in a number of cell types PS serves as the major, or even exclusive, precursor for PE synthesis (see Voelker 1985; Miller \& Kent 1986). The responsible enzyme, PS-decarboxylase (Figure 3 ), has been localized to the outer leaflet of the inner mitochondrial membrane (see Daum 1985; Voelker 1985; Yorek et al 1985). A significant fraction of the cellular PS must thus be transferred from the ER, across the outer membrane to the inner membrane, where it is converted to PE. The $t_{1 / 2}$ of the entire process turned out to be on the order of $5-7 \mathrm{~h}$ (Voelker 1985; Vance \& Vance 1988).${ }^{14}$ The transport of PS was found to be sensitive to metabolic poisons and cycloheximide (Voelker 1985); at present there is no simple explanation for these effects.

PHOSPHATIDYLETHANOLAMINE SYNTHESIS AND TRANSPORT For PE synthesized by decarboxylation of PS in the inner mitochondrial membrane (see above), transport out of the mitochondrion involves three steps: from the inner to the outer membrane (possibly involving the "contact sites"), across the outer membrane, and to the ER or other organelles. The intramitochondrial exchange has been measured to have a rate similar to that of PC, with a $t_{1 / 2}$ of 0.5-3 h (Bygrave 1969; McMurray \& Dawson 1969; Blok et al 1971; Eggens et al 1979); the mechanism of this transport is still unclear. Transport from the outer membrane to the ER, according to the current views, occurs by monomeric exchange through the aqueous phase. This equilibration appears to be much slower than that of PI or PC, with a $t_{1 / 2}$ of about 70 min (McMurray \& Dawson 1969; Blok et al 1971; Yaffe \& Kennedy 1983). A somewhat faster time course has been reported for the equilibration ${ }^{15}$ of newly synthesized PE with PE in the plasma membrane (Sleight \& Pagano 1983; Kobayashi \& Pagano 1989). Their results with various inhibitors and temperatures also are best explained by an exchange

\footnotetext{
${ }^{14}$ Very different time courses have been obtained from radioactive labeling and cell fractionation in yeast (Daum et al 1986) and rat liver (Vance 1988). However, the complexity of the metabolic pathways and the occurrence of multiple pools of phospholipid, the nature of which is unclear at present, complicates evaluations of transport kinetics.

${ }^{15}$ The authors concluded that a rapid inside-outside translocation of PE had taken place across the plasma membrane. In an independent study (see Martin \& Pagano 1987) they demonstrated that C6-NBD-PE and -PS undergo a fast (protein-mediated) translocation in the opposite direction. Also in the erythrocyte membrane PE translocation in both directions is tenfold faster than that of PC (see Zachowski \& Devaux 1989).
} 
process. This conclusion was recently corroborated by the observation that, in contrast to the vesicular transport of sphingolipids, PE transport was not inhibited in mitotic cells (Kobayashi \& Pagano 1989). Although PE like most phospholipids is present in all organelles along the vesicular pathway (Figure 1) and will therefore be shuttled by carrier vesicles, the evidence suggests that monomeric transfer is responsible for intracellular PE equilibration.

It has been suggested (Yorek et al 1985; see Miller \& Kent 1986) that the CDP-ethanolamine pathway on the cytoplasmic surface of the ER and the Golgi (Figure 3, see Jelsema \& Morré 1978; Bell et al 1981; Vance $\&$ Vance 1988) is preferentially used for the synthesis of ethanolamine plasmalogens. Plasmalogens possess a long chain alcohol in ether linkage at the $\mathrm{Cl}$-position of the glycerol, instead of the esterified fatty acid of the regular phospholipids. In addition, the first two carbons of the alcohol are connected by a double bond. They constitute a significant fraction of the cellular phospholipids, especially of the PE of which they routinely make up $50 \%$ or more (see Lazarow 1987; van den Bosch et al 1988). The introduction of the ether bond has been localized to the luminal leaflet of the peroxisomal membrane. All plasmalogens must, therefore, at some stage have passed through this organelle. The mechanism of transport is relatively simple: since acylation of the C2-position of the glycerol and the subsequent steps in plasmalogen biosynthesis only take place at the ER, transport occurs at the alkylglycerophosphate (or alkyl-dihydroxyacetone phosphate) stage. This should present no problem as this lysophosphatidic acid readily exchanges through the aqueous phase (see Bell et al 1981; Lazarow 1987). How the molecule initially translocates to the cytoplasmic leaflet of the peroxisomal membrane is, however, unclear.

MITOCHONDRIAL CARDIOLIPIN (CL) AND LYSOSOMAL LYSOBISPHOSPHATIDIC ACID (LBPA) CL is synthesized in the mitochondrial inner membrane, where the bulk of the CL also resides. It is unclear by what mechanism $\mathrm{CL}$ is retained in the inner membrane, but it appears to be leaky as some $\mathrm{CL}$ is present in the outer membrane (Daum 1985). Phosphatidylglycerol (PG), its precursor, is synthesized in the inner membrane. PG-synthetic activity is also present in ER (see Daum 1985). An answer to the question how this ER-produced PG is handled by the cell may provide new insights into general phospholipid traffic. However, the relative quantity of this PG is very low. Interesting systems, in this respect, are a PG overproducing cell mutant (Esko \& Raetz 1980) and the lung type II cell producing surfactant PG (see Post \& van Golde 1988). The selective presence of LBPA in lysosomes may be explained simply by the fact that it is synthesized there 
(see Bell et al 1981), as in the case of CL in the mitochondria. Interestingly, LBPA was also found in the intracellular vesicles by which Vaccinia virus surrounds its DNA in the process of obtaining its envelope (Hiller et al 1981).

\section{SUMMARY AND PERSPECTIVES}

A central question in the field of the cell biology of lipids is how cells maintain the unique lipid compositions of their organelles. At present, no coherent scheme of intracellular lipid transport can be formulated to account for these differences without numerous assumptions: there is a basic lack of reliable data on the lipid composition of purified subcellular membrane fractions and on the topography of the lipids therein. Part of the solution to this problem must come from defining the site of synthesis, the transbilayer distribution, and the translocation properties of the lipids. A relatively clear case is that of sphingolipids that are assembled somewhere in the Golgi, reside in the exoplasmic leaflet, and do not flip-flop. They can be transported by vesicles only, as they are simply not accessible for cytoplasmic exchange. The situation is more complex for the other lipids, all of which take part, as monomers, in cytoplasmic intermembrane transfer. In the case of cholesterol, a lot may be explained by a preferential interaction with sphingolipids over glycerophospholipids. Also the fact that some lipids, for instance PC, experience flip-flop in some membranes but essentially not in others imposes specificity onto overall transport: Clear areas of research will therefore be to establish the exact site of SPH synthesis, the sidedness (and the flip-flop rate) of cholesterol in cellular membranes, and the intracellular location of lipid transfer proteins. A different set of questions concerns bulk membrane traffic: does the Golgi possess a unidirectional vesicular transport step? How do cells regulate the relative surface areas of their membranes?

Maybe the most exciting direction that emerges from the recent literature is the crucial role of sphingolipids and possibly disaturated glycerophospholipids in sorting processes in the Golgi. By being shuttled exclusively from cis to trans and to the plasmalemma they may be responsible for maintaining the cholesterol gradient in the cell. They are responsible for the establishment of lipid polarity in epithelial cells. As in evolution these processes have evolved coordinately with membrane protein transport, it is expected that both membrane lipids and proteins make up indispensible parts of an intricate sorting machinery. The elucidation of this machinery will mark a breakthrough in molecular membrane biology. 


\section{ACKNOWLEDGMENTS}

I apologize to all researchers to whose original contributions I could only refer via reviews by others. With thanks to Günther Daum, Philippe Devaux, Jerry Faust, Dick Hoekstra, Laura Liscum, Dick Pagano, Konrad Sandhoff, Joachim Seelig, Peter Slotte, Dennis Vance, and Dennis Voelker for opinions, reprints and preprints; to Henk van den Bosch, Arie van der Ende, Wouter van 't Hof, Arie Verkley, Karel Wirtz, and especially to Mark Marsh (London) and Klaas Nicolay for critical comments. I am grateful to them and other colleagues at the Utrecht University for their willingness to discuss what must have seemed at that time to be random issues. The research for this review was sponsored by a senior investigatorship from the Royal Netherlands Academy of Arts and Sciences.

\section{Literature Cited}

Allan, D., Walklin, C. M. 1988. Endovesiculation of human erythrocytes exposed to sphingomyelinase C: a possible explanation for the enzyme-resistant pool of sphingomyelin. Biochim. Biophys. Acta 938: 403-10

Appelkvist, E.-L. 1987. In vitro labeling of peroxisomal cholesterol with radioactive precursors. Biosci. Rep. 7: 853-58

Barenholz, Y., Thompson, T. E. 1980. Sphingomyelins in bilayers and biological membranes. Biochim. Biophys. Acta 604: $129-58$

Barsukov, L. I., Bergelson, L. D., Spiess, M., Hauser, H., Semenza, G. 1986. Phospholipid topology and flip-flop in intestinal brush border membrane. Biochim. Biophys. Acta 862: 87-99

Belcher, J. D., Hamilton, R. L., Brady, S. E., Hornick, C. A., Jaeckle, S., et al. 1987. Isolation and characterization of three endosomal fractions from the liver of estradiol-treated rats. Proc. Natl. Acad. Sci. USA 84: 6785-89

Bell, R. M., Ballas, L. M., Coleman, R. A. 1981. Lipid topogenesis. J. Lipid Res. 22: 391-403

Bishop, W. R., Bell, R. M. 1988. Assembly of phospholipids into cellular membranes: Biosynthesis, transmembrane movement and intracellular translocation. Annu. Rev. Cell Biol. 4: 579-610

Blanchette-Mackie, E. J., Dwyer, N. K., Amende, L. M., Kruth, H. S., Butler, J. D., et al. 1988. Type-C Niemann-Pick disease: low density lipoprotein uptake is associated with premature cholesterol accumulation in the Golgi complex and excessive cholesterol storage in lysosomes. Proc. Natl. Acad. Sci. USA 85: 8022-26
Blok, M. C., Wirtz, K. W. A., Scherphof, G. L. 1971. Exchange of phospholipids between microsomes and inner and outer mitochondrial membranes of rat liver. Biochim. Biophys. Acta 233: 61-75

Bluemink, J. G., van Maurik, P. A. M., Tertoolen, L. G. J., van der Saag, P. T., de Laat, S. W. 1983. Ultrastructural aspects of rapid plasma membrane growth in mitotic neuroblastoma cells. Eur. J. Cell. Biol. 32: 7-16

Brasaemle, D. L., Robertson, A. D., Attie, A. D. 1988. Transbilayer movement of cholesterol in the human erythrocyte membrane. J. Lipid Res. 29: 481-89

Brotherus, J., Renkonen, O. 1977. Phospholipids of subcellular organelles isolated from cultured BHK cells. Biochim. Biophys. Acta 486: 243-53

Burgess, T. L., Kelly, R. B. 1987. Constitutive and regulated secretion of proteins. Annu. Rev. Cell Biol. 3: 243-93

Bygrave, F. L. 1969. Studies on the biosynthesis and turnover of the phospholipid components of the inner and outer membranes of rat liver mitochondria. J. Biol. Chem. 244: 4768-72

Caspar, D. L. D., Kirschner, D. A. 1971. Myelin membrane structure at $10 \AA$ resolution. Nature New Biol. 231: 46-52

Chatterjee, S., Clarke, K. S., Kwiterovich, P. O. Jr. 1986. Regulation of synthesis of lactosylceramide and long chain bases in normal and familial hypercholesterolemic cultured proximal tubular cells. J. Biol. Chem. 261: 13474-79

Chojnacki, T., Dallner, G. 1988. The biological role of dolichol. Biochem. J. 251: 1-9

Cohen, B. G., Phillips, A. H. 1980. Evidence 
for rapid and concerted turnover of membrane phospholipids in MOPC $41 \mathrm{my-}$ eloma cells and its possible relationship to secretion. J. Biol. Chem. 255: 3075-79

Colbeau, A., Nachbaur, J., Vignais, P. M. 1971. Enzymic characterization and lipid composition of rat liver subcellular membranes. Biochim. Biophys. Acta 249: 46292

Cook, H. W., Palmer, F. B. St. C., Byers, D. M., Spence, M. W. 1988. Isolation of plasma membranes from cultured glioma cells and application to evaluation of membrane sphingomyelin turnover. Anal. Biochem. 174: 552-60

Crane, D. I., Masters, C. J. 1986. The effect of clofibrate on the phospholipid composition of the peroxisomal membranes in mouse liver. Biochim. Biophys. Acta 876: 256-63

Daum, G. 1985. Lipids of mitochondria. Biochim. Biophys. Acta 822: 1-42

Daum, G., Heidorn, E., Paltauf, F. 1986. Intracellular transfer of phospholipids in the yeast, Saccharomyces cerevisiae. Biochim. Biophys. Acta 878: 93-101

Davis, P. J., Poznansky, M. J. 1987. Modulation of 3-hydroxy-3-methylglutarylCoA reductase by changes in microsomal cholesterol content or phospholipid composition. Proc. Natl. Acad. Sci. USA 84: 118-21

Dawidowicz, E. A. 1987. Dynamics of membrane lipid metabolism and turnover. Annu. Rev. Biochem. 56: 43-61

DeGrella, R. F., Simoni, R. D. 1982. Intracellular transport of cholesterol to the plasma membrane. J. Biol. Chem. 257: 14256-62

Demel, R. A., Jansen, J. W. C. M., van Dijck, P. W. M., van Deenen, L. L. M. 1977. The preferential interaction of cholesterol with different classes of phospholipids. Biochim. Biophys. Acta 465: 1-10

De Silva, N. S., Siu, C.-H. 1981. Vesiclemediated transfer of phospholipids to plasma membrane during cell aggregation of Dictyostelium discoideum. J. Biol. Chem. 256: $5845-50$

Devaux, P. F. 1988. Phospholipid flippases. FEBS Lett. 234: 8-12

Devaux, P. F., Seigneuret, M. 1985. Specificity of lipid-protein interactions as determined by spectroscopic techniques. Biochim. Biophys. Acta 822: 63-125

Dickson, R. B., Beguinot, L., Hanover, J. A., Richert, N. D., Willingham, M. C., Pastan, I. 1983. Isolation and characterization of a highly enriched preparation of receptosomes (endosomes) from a human cell line. Proc. Natl. Acad. Sci. US A 80: 5335--39

Dyatlovitskaya, E. V., Timofeeva, N. G.,
Yakimenko, E. F., Barsukov, L. I., Muzya, G. I., Bergelson, L. D. 1982. A sphingomyelin transfer protein in rat tumors and fetal liver. Eur. J. Biochem. 123: $311-5$

Eggens, I., Valtersson, C., Dallner, G., Ernster, L. 1979. Transfer of phospholipids between the Endoplasmic Reticulum and mitochondria in rat hepatocytes in vivo. Biochem. Biophys. Res. Commun. 91: 70914

Esko, J. D., Raetz, C. R. H. 1980. Mutants of Chinese hamster ovary cells with altered membrane phospholipid composition. Replacement of phosphatidylinositol by phosphatidylglycerol in a myoinositol auxotroph. J. Biol. Chem. 255: 4474-80

Evans, W. H., Hardison, W. G. M. 1985. Phospholipid, cholesterol, polypeptide and glycoprotein composition of hepatic endosome subf ractions. Biochem. J. 232: 33-36

Farquhar, M. G. 1985. Progress in unraveling pathways of Golgi traffic. Annu. Rev. Cell Biol. 1: 447-88

Fishman, P. H., Bradley, R. M., Hom, B. E., Moss, J. 1983. Uptake and metabolism of exogenous gangliosides by cultured cells: effect of choleragen on the turnover of $\mathrm{G}_{\mathrm{MI}}$. J. Lipid Res. 24: 1002-11

Fuchs, R., Mâle, P., Mellman, I. 1989. Acidification and ion permeabilities of highly purified rat liver endosomes. J. Biol. Chem. 264: 2212-20

Fujiki, Y., Fowler, S., Shio, H., Hubbard, A. L., Lazarow, P. B. 1982. Polypeptide and phospholipid composition of the membrane of rat liver peroxisomes: comparison with Endoplasmic Reticulum and mitochondrial membranes. J. Cell Biol 93: 103-10

Fürst, W., Machleidt, W., Sandhoff, K. 1988. The precursor of sulfatide activator protein is processed to three different proteins. Biol. Chem. Hoppe-Se yler 369: $317-$ 28

Gardam, M. A., Itovitch, J. J., Silvius, J. R. 1989. Partitioning of exchangeable fluorescent phospholipids and sphingolipids between different lipid bilayer environments. Biochemistry 28: 884-93

Gueze, H. J., Slot, J. W., Schwartz, A. L. 1987. Membranes of sorting organelles display lateral heterogeneity in receptor distribution. J. Cell Biol. 104: 1715-23

Griffiths, G., Simons, K. 1986. The trans Golgi network: sorting at the exit site of the Golgi complex. Science 234: 438-43

Hansson, G. C., Simons, K., van Meer, G. 1986. Two strains of the Madin-Darby canine kidney (MDCK) cell line have distinct glycosphingolipid compositions. EMBO J. 5: 483-89 
Helmkamp, G. M. Jr. 1986. Phospholipid transfer proteins: mechanism of action. $J$. Bioenerg. Biomembr. 18: 71-91

Helmy, S., Porter-Jordan, K., Dawidowicz, E. A., Pilch, P., Schwartz, A. L., Fine, R. E. 1986. Separation of endocytic from exocytic coated vesicles using a novel cholinesterase mediated density shift technique. Cell 44: 497-506

Henning, R., Stoffel, W. 1973. Glycosphingolipids in lysosomal membranes. Hoppe-Seyler's Z. Physiol. Chem. 354: 760-70

Hiller, G., Eibl, H., Weber, K. 1981. Acyl bis(monoacylglycero)phosphate, assumed to be a marker for lysosomes, is a major phospholipid of Vaccinia virions. Virology 113: 761-64

Jelsema, C. L., Morré, D. J. 1978. Distribution of phospholipid biosynthetic enzymes among cell components of rat liver. J. Biol. Chem. 253: 7960-71

Kaplan, M. R., Simoni, R. D. 1985a. Intracellular transport of phosphatidylcholine to the plasma membrane. J. Cell Biol. 101: $441-45$

Kaplan, M. R., Simoni, R. D. 1985b. Transport of cholesterol from the Endoplasmic Reticulum to the plasma membrane. $J$. Cell Biol. 101: 446-53

Keenan, T. W., Morré, D. J. 1970. Phospholipid class and fatty acid composition of Golgi apparatus isolated from rat liver and comparison with other cell fractions. Biochemistry 9: 19-25

Keenan, T. W., Huang, C. M., Morré, D. J. 1972. Gangliosides: Non-specific localization in the surface membranes of bovine mammary gland and rat liver. Biochem. Biophys. Res. Comm. 47: $1277-$ 83

Klein, D., Leinekugel, P., Pohlentz, G., Schwarzmann, G., Sandhoff, K. 1988. Metabolism and intracellular transport of gangliosides in cultured fibroblasts. In New Trends in Ganglioside Research: Neurochemical and Neuroregenerative Aspects. eds. R. W. Leeden, E. L. Hogan, G. Tettamanti, A. J. Yates, R. K. Yu, pp. 247-58 Padova: Liviana

Kobayashi, T., Pagano, R. E. 1989. Lipid transport during mitosis: alternative pathways for delivery of newly synthesized lipids to the cell surface. J. Biol. Chem. 264: 5966-73

Koval, M., Pagano, R. E. 1989. Lipid recycling between the plasma membrane and intracellular compartments: transport and metabolism of fluorescent sphingomyelin analogs in cultured fibroblasts. $J$. Cell Biol. In press

Kuge, O., Nishijima, M., Akamatsu, Y. 1986. Phosphatidylserine biosynthesis in cultured Chinese hamster ovary cells. III. Genetic evidence for utilization of phosphatidylcholine and phosphatidylethanolamine as precursors. J. Biol. Chem. 261: 5795-98

Lambeth, J. D., Xu, X. X., Glover, M. 1987. Cholesterol sulfate inhibits adrenal mitochondrial cholesterol side chain cleavage at a site distinct from cytochrome $\mathrm{P}-450_{\text {scc. }}$ Evidence for an intramitochondrial cholesterol translocator. J. Biol. Chem. 262: 9181-88

Lange, Y., D'Alessandro, J. S., Small, D. M. 1979. The affinity of cholesterol for phosphatidylcholine and sphingomyelin. Biochim. Biophys. Acta 556: 388-98

Lange, Y., Matthies, H. J. G. 1984. Transfer of cholesterol from its site of synthesis to the plasma membrane. J. Biol. Chem. 259: 14624-30

Lange, Y., Muraski, M. F. 1988. Topographic heterogeneity in cholesterol biosynthesis. J. Biol. Chem. 263: 936673

Lange, Y., Swaisgood, M. H., Ramos, B. V., Steck, T. L. 1989. Plasma membranes contain half the phospholipid and $90 \%$ of the cholesterol and sphingomyelin in cultured human fibroblasts. J. Biol. Chem. 264: 3786-93

Lazarow, P. B. 1987. The role of peroxisomes in mammalian cellular metabolism. $J$. Inher. Metab. Dis. (10 Suppl.) 1: 11-22

Lipsky, N. G., Pagano, R. E. 1985. Intracellular translocation of fluorescent sphingolipids in cultured fibroblasts: endogenously synthesized sphingomyelin and glucocerebroside analogues pass through the Golgi apparatus en route to the plasma membrane. J. Cell Biol. 100: 27-34

Liscum, L., Faust, J. R. 1987. Low density lipoprotein (LDL)-mediated suppression of cholesterol synthesis and LDL uptake is defective in Niemann-Pick type C fibroblasts. J. Biol. Chem. 262: 17002-8

Liscum, L., Ruggiero, R. M., Faust, J. R. 1989. The intracellular transport of LDLderived cholesterol is defective in Niemann-Pick, type C fibroblasts. J. Cell Biol. 108: 1625-36

Luzio, J. P., Stanley, K. K. 1983. The isolation of endosome-derived vesicles from rat hepatocytes. Biochem. J. 216: 27-36

Marggraf, W. D., Anderer, F. A., Kanfer, J. N. 1981. The formation of sphingomyelin from phosphatidylcholine in plasma membrane preparations from mouse fibroblasts. Biochim. Biophys. Acta 664: 61-73

Martin, O. C., Pagano, R. E. 1987. Transbilayer movement of fluorescent analogs of phosphatidylserine and phosphatidylethanolamine at the plasma membrane of cultured cells. Evidence for a 
protein-mediated and ATP-dependent process(es). J. Biol. Chem. 262: 5890-98

Matlin, K. S. 1986. The sorting of proteins to the plasma membrane in epithelial cells. J. Cell Biol. 103: 2565-68

McMurray, W. C., Dawson, R. M. C. 1969. Phospholipid exchange reactions within the liver cell. Biochem. J. 112: 91-108

Miller, M. A., Kent, C. 1986. Characterization of the pathways for phosphatidylethanolamine biosynthesis in Chinese hamster ovary mutant and parental cell lines. J. Biol. Chem. 261: 975361

Miller-Prodraza, H., Fishman, P. H. 1984. Effect of drugs and temperature on biosynthesis and transport of glycosphingolipids in cultured neurotumor cells. Biochim. Biophys. Acta 804: 44-51

Mills, J. T., Furlong, S. T., Dawidowicz, E. A. 1984. Plasma membrane biogenesis in eukaryotic cells: translocation of newly synthesized lipid. Proc. Natl. Acad. Sci. USA 81: 1385-88

Neefjes, J. J., Verkerk, J. M. H., Broxterman, H. J. G., van der Marel, G. A., van Boom, J. H., Ploegh, H. L. 1988. Recycling glycoproteins do not return to the cis-Golgi. J. Cell Biol. 107: 79-87

O'Brien, J. S., Kretz, K. A., Dewji, N., Wenger, D. A., Esch, F., Fluharty, A. L. 1988. Coding of two sphingolipid activator proteins (SAP-1 and SAP-2) by the same genetic locus. Science 241: 1098-101

Op den Kamp, J. A. F. 1979. Lipid asymmetry in membranes. Annu. Rev. Biochem. 48: 47-71

Orci, L., Montesano, R., Meda, P., Malaisse-Lagae, F., Brown, D., et al. 1981. Heterogeneous distribution of filipincholesterol complexes across the cysternae of the Golgi apparatus. Proc. Natl. Acad. Sci. USA 78: 293-7

Orci, L., Brown, M. S., Goldstein, J. L., Garcia-Segura, L. M., Anderson, R. G. W. 1984. Increase in membrane cholesterol: a possible trigger for degradation of HMG CoA reductase and crystalloid Endoplasmic Reticulum in UT-1 cells. Cell 36: 835-45

Pagano, R. E. 1988. What is the fate of diacylglycerol produced at the Golgi apparatus? TIBS 13: 202-5

Pagano, R. E., Sleight, R. G. 1985. Defining lipid transport pathways in animal cells. Science 229: 1051-57

Phillips, M. C., Johnson, W. J., Rothblat, G. H. 1987. Mechanisms and consequences of cellular cholesterol exchange and transfer. Biochim. Biophys. Acta 906: 223-76

Pierce, M., Turley, E. A., Roth, S. 1980. Cell surface glycosyltransferase activities. Int . Rev. Cytol. 65: 1-47
Post, M., van Golde, L. M. G. 1988. Metabolic and developmental aspects of the pulmonary surfactant system. Biochim. Biophys. Acta 947: 249-86

Reinhart, M. P., Billheimer, J. T., Faust, J. R., Gaylor, J. L. 1987. Subcellular localization of the enzymes of cholesterol biosynthesis and metabolism in rat liver. J. Biol. Chem. 262: 9649-55

Robertson, D. L., Poznansky, M. J. 1985. The effect of non-receptor-mediated uptake of cholesterol on intracellular cholesterol metabolism in human skin fibroblasts. Biochem. J. 232: 553-57

Rothman, J. E. 1981. The Golgi apparatus: Two organelles in tandem. Science 213: 1212-19

Rousselet, A., Colbeau, A., Vignais, P. M., Devaux, P. F. 1976. Study on the transverse diffusion of spin-labeled phospholipids in biological membranes. II. Inner mitochondrial membrane of rat liver: use of phosphatidylcholine exchange protein. Biochim. Biophys. Acta 426: 37284

Salas, P. J. I., Vega-Salas, D. E., Hochman, J., Rodriguez-Boulan, E., Edidin, M. 1988. Selective anchoring in the specific plasma membrane domain: a role in epithelial cell polarity. J. Cell Biol. 107: 236376

Sandra, A., Pagano, R. E. 1978. Phospholipid asymmetry in LM cell plasma membrane derivatives: polar head group and acyl chain distributions. Biochemistry 17: $332-38$

Scallen, T. J., Pastuszyn, A., Noland, B. J., Chanderbhan, R., Kharroubi, A., Vahouny, G. V. 1985. Sterol carrier and lipid transfer proteins. Chem. Phys. Lip. 38: 239-61

Sheetz, M. P., Singer, S. J. 1974. Biological membranes as bilayer couples. A molecular mechanism of drug-erythrocyte interactions. Proc. Natl. Acad. Sci. USA 71: 4457-61

Simons, K., Fuller, S. D. 1985. Cell surface polarity in epithelia. Annu. Rev. Cell Biol. 1: 243-88.

Simons, K., van Meer, G. 1988. Lipid sorting in epithelial cells. Biochemistry 27: 6197202

Sleight, R. G., Pagano, R. E. 1983. Rapid appearance of newly synthesized phosphatidylethanolamine at the plasma membrane. J. Biol. Chem. 258: 9050-58

Slotte, J. P., Bierman, E. L. 1987. Movement of plasma-membrane sterols to the endoplasmic reticulum in cultured cells. Biochem. J. 248: 237-42

Slotte, J. P., Bierman, E. L. 1988. Depletion of plasma membrane-sphingomyelin rapidly alters the distribution of cholesterol 
between plasma membranes and intracellular cholesterol pools in cultured fibroblasts. Biochem. J. 250: 653-58

Slotte, J. P., Oram, J. F., Bierman, E. L. 1987. Binding of high density lipoproteins to cell receptors promotes translocation of cholesterol from intracellular membranes to the cell surface. J. Biol. Chem. 262: 12904-7

Steck, T. L., Kezdy, F. J., Lange, Y. 1988. An activation-collision mechanism for cholesterol transfer between membranes. J. Biol. Chem. 263: 13023-31

Sune, A., Vidal, M., Morin, P., SainteMarie, J., Bienvenue, A. 1988. Evidence for bidirectional transverse diffusion of spin-labeled phospholipids in the plasma membrane of guinea pig blood cells. Biochim. Biophys. Acta 946: 315-27

Symington, F. W., Murray, W. A., Bearman, S. I., Hakomori, S.-i. 1987. Intracellular localization of lactosylceramide, the major human neutrophil glycosphingolipid. $J$. Biol. Chem. 262: 11356-63

Tanaka, N., Leduc, E. H. 1956. A study of the cellular distribution of Forssman antigen in various species. J. Immunol. 77: 198-212

Thompson, S. L., Burrows, R., Laub, R. J., Krisans, S. K. 1987. Cholesterol synthesis in rat liver peroxisomes. J. Biol. Chem. 262: $17420-25$

Thompson, T. E., Tillack, T. W. 1985. Organization of glycosphingolipids in bilayers and plasma membranes of mammalian cells. Annu. Rev. Biophys. Biophys. Chem. 14: 361-86

Tsuneoka, M., Yamamoto, A., Fujiki, Y., Tashiro, Y. 1988. Nonspecific lipid transfer protein (sterol carrier protein-2) is located in rat liver peroxisomes. $J$. Biochem. 104: 560-64

Urade, R., Hayashi, Y., Kito, M. 1988. Endosomes differ from plasma membranes in the phospholipid molecular species composition. Biochim. Biophys. Acta 946: 151-63

van Amerongen, A., van Noort, M., van Beckhoven, J. R. C. M., Rommerts, F. F. G., Orly, J., Wirtz, K. W. A. 1989. The subcellular distribution of the non-specific lipid transfer protein (sterol carrier protein 2 ) in rat liver and adrenal gland. Biochim. Biophys. Acta 1001: 243-48

Vance, J. E. 1988. Compartmentalization and differential labeling of phospholipids of rat liver subcellular membranes. Biochim. Biophys. Acta 963: 10-20

Vance, D. E., Ridgway, N. D. 1988. The methylation of phosphatidylethanolamine. Prog. Lipid Res. 27: 61-79

Vance, J. E., Vance, D. E. 1988. Does rat liver Golgi have the capacity to synthesize phospholipids for lipoprotein secretion. $J$. Biol. Chem. 263: 5898-909

van den Bosch, H., Schalkwijk, C. G., Schrakamp, G., Wanders, R. J. A., Schutgens, R. B. H., et al. 1988. Aberration in de novo ether lipid biosynthesis in peroxisomal disorders. In Biological Membranes: Aberrations in Membrane Structure and Function eds. M. L. Karnovsky, A. Leaf, L. C. Bolis, pp. 139-50, New York: Liss

van Meer, G. 1986. The lipid bilayer of the ER. TIBS 11: 194-95; 401

van Meer, G. 1987. Plasma membrane cholesterol pools. TIBS 12: 375-76

van Meer, G. 1988. How epithelial cells grease their microvilli. TIBS 13: 242-43

van Meer, G., Simons, K. 1986. The function of tight junctions in maintaining differences in lipid composition between the apical and the basolateral cell surface domains of MDCK cells. EMBO J. 5: 1455-64

van Meer, G., Stelzer, E. H. K., Wijnaendtsvan-Resandt, R. W., Simons, K. 1987. Sorting of sphingolipids in epithelial (Madin-Darby canine kidney) cells. J. Cell Biol. 105: 1623-35

Van Paridon, P. A., Gadella, T. W. J. Jr., Somerhar ju, P. J., Wirtz, K. W. A. 1987. On the relationship between the dual specificity of the bovine brain phosphatidylinositol transfer protein and membrane phosphatidylinositol levels. Biochim. Biophys. Acta 903: 68-77

Venien, C., Le Grimellec, C. 1988. Phospholipid asymmetry in renal brush-border membranes. Biochim. Biophys. Acta 942: 159-68

Voelker, D. R. 1985. Disruption of phosphatidylserine translocation to the mitochondria in baby hamster kidney cells. $J$. Biol. Chem. 260: 14671-76

Voelker, D. R., Frazier, J. L. 1986. Isolation and characterization of a Chinese hamster ovary cell line requiring ethanolamine or phosphatidylserine for growth and exhibiting defective phosphatidylserine synthase activity. J. Biol. Chem. 261: 1002-8

Voelker, D. R., Kennedy, E. P. 1982. Cellular and enzymic synthesis of sphingomyelin. Biochemistry 21: 2753-9

Wattenberg, B. W., Silbert, D. F. 1983. Sterol partitioning among intracellular membranes. Testing a model for cellular sterol disribution. J. Biol. Chem. 258: 2284-89

Weinstein, D. B., Marsh, J. B., Glick, M. C., Warren, L. 1970. Membranes of animal cells. VI. The glycolipids of the L cell and its surface membrane. J. Biol. Chem. 245: 3928-37

Wherrett, J. R., Huterer, S. 1972. Enrich- 
ment of bis-(monoacylglyceryl) phosphate in lysosomes from rat liver. J. Biol. Chem. 247: 4114-20

Wieland, F. T., Gleason, M. L., Serafini, T. A., Rothman, J. E. 1987. The rate of bulk flow from the Endoplasmic Reticulum to the cell surface. Cell 50: 289-300

Yaffe, M. P., Kennedy, E. P. 1983. Intracellular phospholipid movement and the role of phospholipid transfer proteins in animal cells. Biochemistry 22: $1497-$ 507

Yeagle, P. L. 1985. Cholesterol and the cell membrane. Biochim. Biophys. Acta 822: 267-87
Yorek, M. A., Rosario, R. T., Dudley, D. T., Spector, A. A. 1985. The utilization of ethanolamine and serine for ethanolamine phosphoglyceride synthesis by human Y79 retinoblastoma cells. J. Biol. Chem. 260: 2930-36

Zachowski, A., Devaux, P. F. 1989. Bilayer asymmetry and lipid transport across biomembranes. Comm. Mol. Cell. Biophys. In press

Zambrano, F., Fleischer, S., Fleischer, B. 1975. Lipid composition of the Golgi apparatus of rat kidney and liver in comparison with other subcellular organelles. Biochim. Biophys. Acta 380: 357-69 